BULL. AUSTRAL. MATH. SOC.

\title{
THE GRAVITATIONAL LENS EFFECT \\ OF GALAXIES AND BLACK HOLES
}

\section{IGOR BRAY}

This thesis examines the multi-imaging aspect of the gravitational lens effect of both galaxies and black holes. Galaxies are modeled by spheroids, the density of which is assumed to have spheroidal symmetry, that is, the density is constant on the surface as well as all the other spheroidal shells that make up the spheroid. If such a spheroid is projected onto a plane then the resultant solid ellipse's density has elliptical symmetry.

It is shown that the equation $z=z_{0}-4 D G c^{-2} I^{\star}\left(z_{0}\right) \quad$ which relates the position of an image $z_{0}$ with the position of the source $z$, must satisfy certain symuetry properties. In paxticular,

$$
z=z_{0}-4 D G c^{-2} I^{*}\left(z_{0}\right) \Rightarrow-z=-z_{0}-4 D G c^{-2} I^{*}\left(-z_{0}\right) .
$$

The solution for $I\left(z_{0}\right)$ presented by Bourassa and Kantowski [1] violates this property, as their $I\left(z_{0}\right)=I\left(-z_{0}\right)$. Thus a corrected $I\left(z_{0}\right)$ is computed. This contains a proof of the fact that if the projected density has elliptical symmetry, then there is no contribution to the bending angle due to mass lying outside the ellipse formed by the impact

Received 27 August 1986. Thesis submitted to University of Adelaide February 1986. Degree approved August 1986. Supervisor:: Dr. P. Szekeres.

Copyright Clearance Centre, Inc. Serial-fee code: 0004-9727/87 $\$ A 2.00+0.00$. 
parameter. The new $I\left(z_{0}\right)$ is used to present diagrams which show how many images of a single source will result as a function of the position of the source with respect to the galaxy.

Similar diagrams are presented for the case where it is the Kerr black hole that behaves as a gravitational lens. Unlike other massive objects black holes have $r$ = constant orbits for null geodesics. This implies that a single source of light will have infinitely many images at the observer. These consist of the direct rays and rays which orbit the black hole once, twice, etcetera. We concern ourselves with the direct rays as these are the ones which are least deviated, hence least deformed and most intense, and therefore, are the ones which are most likely to be observed.

Approximate solutions to the equations of motion for direct null geodesics in the Kerr metric are derived. These are correct up to and including second order terms in $m / r_{\min }$ and $a / r_{\min }$, where $m$ is the Kerr mass, $a$ is the Kerr spin and $r_{\min }$ the distance of closest approach, and are sufficient for most astronomical purposes. Second order terms are included as spin comes in only at this order and our primary interest is the effect of spin on image multiplicity. It is found that the derived expressions can be combined with numerical integration to give excellent results for rays which have relatively large deviations.

\section{References}

[1] Eourassa, R.R. and Kantowski, R., Astrophys. J. 13 (1975) 195.

School of Physical Sciences,

The Flinders University of South Australia,

Bedford Park, 5042

South Australia,

Australia. 